\title{
Microwave Support of the Alcoholic Fermentation Process of Cyanobacteria Arthrospira platensis ${ }^{+}$
}

\author{
Anna Nowicka *, Marcin Zieliński and Marcin Dębowski \\ Department of Environment Engineering, University of Warmia and Mazury in Olsztyn, \\ Warszawska St. 117a, 10-720 Olsztyn, Poland; marcin.zielinski@uwm.edu.pl (M.Z.); \\ marcin.debowski@uwm.edu.pl (M.D.) \\ * Correspondence: anna.grala@uwm.edu.pl; Tel.: +48-89-523-3235 \\ + Presented at Environment, Green Technology and Engineering International Conference (EGTEIC 2018), \\ Caceres, Spain, 18-20 June 2018.
}

Published: 22 October 2018

\begin{abstract}
The aim of this study was to determine the effect of thermal hydrolysis on degradation of polysaccharides contained in biomass of cyanobacteria Arthrospira platensis and to assess the effectiveness of ethanol production from pre-conditioned biomass. The study aimed at the selection of the most advantageous parameters of thermo hydrolysis to reach the experiment variant with the best effects, effectiveness of alcohol fermentation. The experiment was divided into two stages; in stage I, the possibility of obtaining fermentable sugars by hydrothermal and treatment of the substrate was tested. Stage II involved an assessment of the effectiveness of the pretreatment methods to produce bioethanol in alcohol fermentation. Yeast used in industrial ethanol production-Saccharomyces cerevisiae As4-was used in the alcohol fermentation. The results have shown that the temperature of $150^{\circ} \mathrm{C}$ was the most beneficial for the process of thermohydrolysis. The control substrate yielded $0.49 \mathrm{~g} / \mathrm{L}$ of bioethanol, the alcohol concentration in the conventionally pre-tretment sample increased by $45 \%$, i.e., to $0.71 \mathrm{~g} / \mathrm{L}$, the mash in the microwave-treatment sample contained the highest concentration of alcohol: $0.97 \mathrm{~g} / \mathrm{L}$, which is $98 \%$ more than in the control mash and $37 \%$ more than in the conventionally heated sample.
\end{abstract}

Keywords: bioethanol; Arthrospira platensis; pretreatment; microwave

\section{Introduction}

Fuels obtained from renewable sources can be divided into first, second and third generation biofuels. Biofuel production from raw materials also used for food production (first generation biofuels) has negative economic consequences, such as an increase in food prices and decreasing area of forests which are replaced by arable land. Alternatively, biofuels can be obtained from other raw materials, such as plant biomass and waste. In terms of quantity, materials containing lignocellulosic complex, such as straw, wood and plant waste from food production, account for the majority of raw materials in the production of second generation biofuels. Development of renewable energy sources is associated with the concept of the third generation fuels, which include algae and aquatic plants; according to numerous studies, aquatic biomass can yield biogas, bioethanol and biodiesel. Pretreatment is of key importance for ensuring a high output of monosaccharides from the polysaccharides present in the material under treatment. Without the pretreatment stage, hydrolysis efficiency of less than $20 \%$ can be achieved, while pretreatment can increase it to $90 \%$ and above [1]. Physical, physico-chemical, chemical and biological pretreatment is conducted in order to increase the efficiency of energy production from biomass. It is important to establish the technological conditions which ensure that the conditioning process will be efficient, cost-effective and safe. If the treatment is not sufficiently effective, it can result in the formation of 
toxic compounds which inhibit the metabolism of methanogenic bacteria [2]. To increase the productivity of ethanol fermentation, microwave pretreatment was tested in this study. Considering the effects of changes of lignocellulosic complex ultrastructure, microwave-based heating can be an alternative to conventional heating (Deng et al. 2015). Microwave radiation delignifies and partly removes hemicellulose and increases hydrolysis of sugars. Conventional heating is based on surface heat exchange; with microwaves, heat is generated by the electromagnetic field. Microwave radiation destroys cellulose by molecular collisions caused by dielectric polarization. The advantages of this method include: the short duration of the process, high selectivity and a smaller amount of the energy supplied compared to conventional heating [1].

The aim of this study was to determine the effect of thermal hydrolysis on degradation of polysaccharides contained in biomass of cyanobacteria A. Platensis and to assess the effectiveness of ethanol production from pre-conditioned biomass. The effect of microwave-based and conventional heating on bioethanol production was compared.

\section{Materials and Methods}

Biomass of $A$. platensis obtained by the authors was used as the study material. The experiment was divided into two stage, I, hydrothermal treatment of the substrate was tested. Stage II involved an assessment of the effectiveness of the pretreatment methods to produce bioethanol in alcohol fermentation. The first stage of the study, with pretreatment, involved simultaneous testing of two variants of heating: microwave-based and conventional; each variant of the experiment was tested in three series at a different process temperature. The hydrothermal pretreatment was conducted for 20 min in both heating at $150{ }^{\circ} \mathrm{C}$. Thermohydrolysis was conducted in two heating technologies. The hydrothermal microwave treatment was carried out with the Mars-Solvent Extraction system. Conventional heating of the plant material was done with a four-stand Laboplay O420E oil bath, in which silicon oil was used as the heating agent. The conditions during the thermohydrolysis - both with conventional and microwave-based heating-were the same. In stage two alcohol fermentation was conducted with an industrial yeast strain used in the industrial production of ethanol: Saccharomyces cerevisiae As4. Inoculum of yeast S. cerevisiae was done with liquid YPG medium: $10 \mathrm{~g}$ of yeast extract, $20 \mathrm{~g}$ of peptone, $20 \mathrm{~g}$ of glucose and $20 \mathrm{~g}$ agar dissolved in $1000 \mathrm{~cm}^{3}$ of distilled water. The $\mathrm{pH}$ was brought to 5.2 by adding $1 \mathrm{M} \mathrm{HCl}$ and controlled by a universal $\mathrm{pH}$-meter. $5 \mathrm{~cm}^{3}$ of sterile distilled water was transferred into a tube with a yeast culture on YPG and washout was performed. The cell suspension obtained in the process was introduced to a conical flask with 100 $\mathrm{cm}^{3}$ of liquid YPG medium and incubated for $24 \mathrm{~h}$ in a shaker at a temperature of $30^{\circ} \mathrm{C}$.

Alcohol fermentation was conducted in an SHF (Separate Hydrolysis and Fermentation) system. After hydrolysis, the substrate was fermented. The inoculum of the appropriate yeast strain was added to the prepared medium (substrate after pretreatment). Fermentation was conducted for $72 \mathrm{~h}$ under anaerobic conditions, at an appropriate temperature for the yeast strain, i.e., at $38^{\circ} \mathrm{C}$. The content of ethanol in the medium was determined after the fermentation was completed. This was done by the method using Hach Lange's LCK 300 reagents. Stage II involved testing three variants of the experiment tested at stage I. The variant was tested in which substrate pretreatment was conducted with microwave radiation at the most beneficial temperature; likewise, alcohol fermentation was conducted for substrates prepared in the same manner for pretreatment with conventional heating. The third variant under test was a control sample, i.e., a substrate without any pretreatment.

\section{Results and Discussion}

The content of ethanol in the medium-"mash" - was determined after fermentation was completed $(72 \mathrm{~h})$. Fermented samples were prepared at $150{ }^{\circ} \mathrm{C}$. The control substrate yielded $0.49 \mathrm{~g} / \mathrm{L}$ of bioethanol, the alcohol concentration in the conventionally pre-heated sample increased by $45 \%$, i.e., to $0.71 \mathrm{~g} / \mathrm{L}$, the microwave-heated sample contained the highest concentration of alcohol: 0.97 $\mathrm{g} / \mathrm{L}$, which is more by $98 \%$ than in the control mash and more by $37 \%$ than in the conventionally heated sample (Figure 1). 


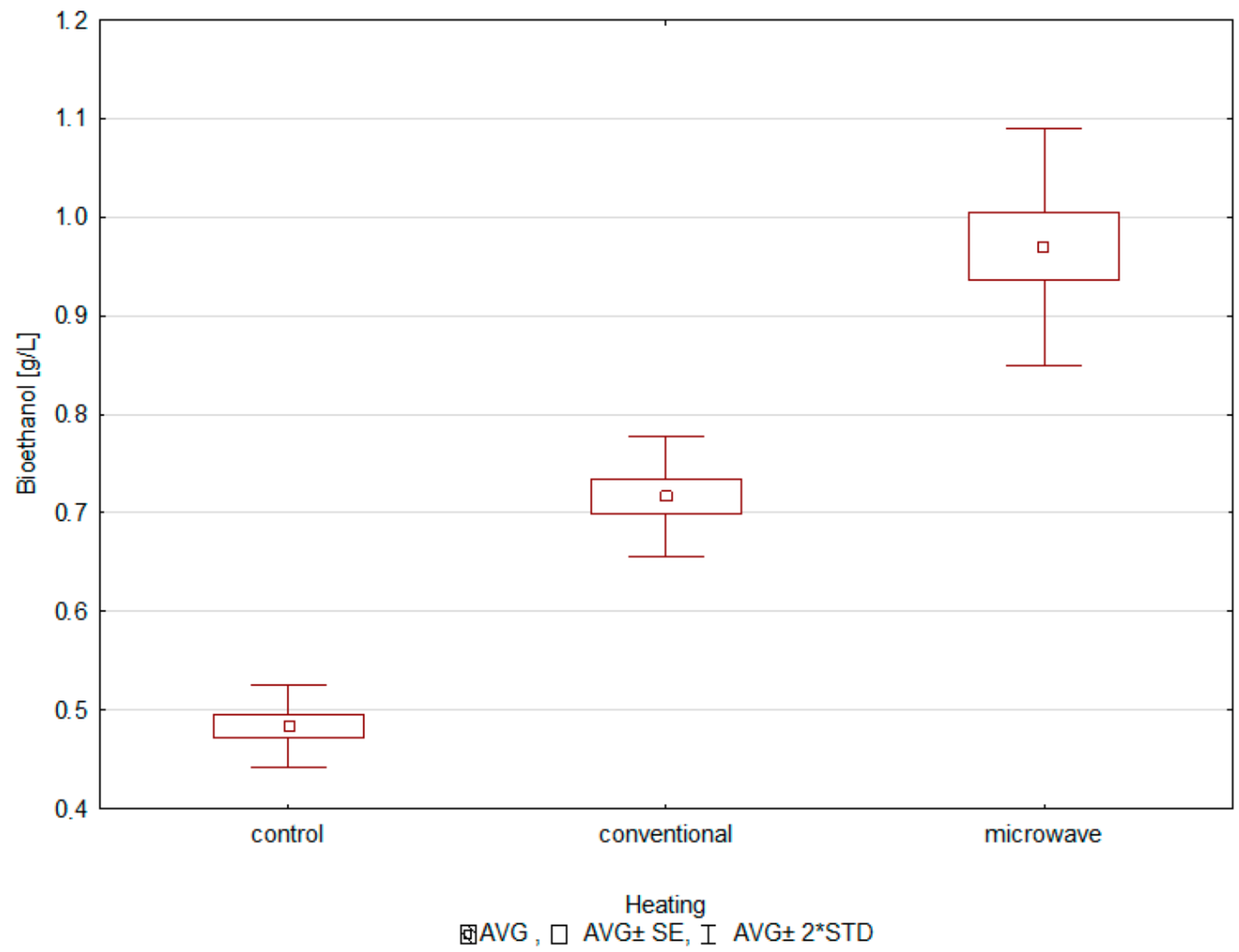

Figure 1. Concentration of bioethanol in relation to the type of thermohydrolysis.

The literature provides examples of application of microwaves as a conditioning factor in alcohol fermentation. Substrate exposure to microwave radiation in various temperature ranges has shown that microwaves increase solubilisation of substrates and break the network of exopolysaccharides, thereby speeding up chemical processes [1]. Klein et al. (2016) applied microwave radiation to increase the production of bioethanol from leaves of sacred fig. The authors applied simultaneous microwave-chemical treatment with hydrochloric acid as the reagent used in the experiment. They compared the results with conventionally heated samples. They tested saccharification of $1 \mathrm{~g}$ of the substrate with $20 \mathrm{~mL}$ of $\mathrm{HCl}$ as a function of acid concentration, time and temperature of exposure to microwave radiation. They observed the most satisfying results after adding $1 \mathrm{M} \mathrm{HCl}$ to the sacred fig leaves, followed by microwave heating for $8 \mathrm{~min}$ at temperatures increasing from 80 to $110{ }^{\circ} \mathrm{C}$. The application of microwave radiation increased glucose production by up to $246 \%$ compared to conventionally heated samples. After the pretreatment, alcohol fermentation of the hydrolysate was conducted with Saccharomyces cerevisiae; the maximum ethanol recovery was $3 \%(w / w)$ of the dry weight of the substrate used [3]. Microwave thermohydrolysis conducted by those authors increased the amount of bioethanol production by 98\% compared to the control sample. Meinita et al. (2013) examined production of bioethanol from red algae: Gelidium amansii, Gracilaria tenuistipitata and Gracilariopsis chorda. The algae were hydrolysed at $130{ }^{\circ} \mathrm{C}$ for $15 \mathrm{~min}$ with $0.2 \mathrm{M} \mathrm{H}_{2} \mathrm{SO}_{4}$ and subsequently fermented at $30^{\circ} \mathrm{C}$; the distillate contained from 0.5 to $0.83 \mathrm{~g} / \mathrm{L}$ of ethanol [4]. Wu et al. (2014) conducted fermentation of algae of Gracilaria sp. and produced ethanol with the yeast Saccharomyces cerevisiae Wu-Y2; they obtained maximally $4.72 \mathrm{~g} / \mathrm{L}$ of ethanol from $11.85 \mathrm{~g} / \mathrm{L}$ of glucose [5]. 


\section{Conclusions}

The application of microwave heating with a view to hydrothermal disintegration of cyanobacteria A.platensis helped to achieve higher efficiency of glucose release and production of ethanol compared to conventional heating. The application of microwave technology increased the amount of produced glucose by a maximum of $62 \%$ compared a sample with no pretreatment. The results have shown that $150{ }^{\circ} \mathrm{C}$ was the most beneficial for the thermohydrolysis process, both in the microwave and conventional heating. The results show that the increased concentration of phenolic compounds did not inhibit alcohol fermentation; the control mash yielded $0.49 \mathrm{~g} / \mathrm{L}$ of bioethanol, the alcohol concentration in the conventionally pre-heated sample increased by $45 \%$ (to $0.71 \mathrm{~g} / \mathrm{L}$ ), the mash in the microwave-heated sample contained the highest concentration of alcohol: $0.97 \mathrm{~g} / \mathrm{L}$, which is $98 \%$ more than in the control mash and $37 \%$ more than in the conventionally heated sample.

Author Contributions: M.Z., M.D., M.H. and E.K. conceived and designed the experiments; P.R. and E.A. performed the experiments; M.Z., M.D. and P.R. analyzed the data; P.R. wrote the paper.

Acknowledgments: This paper was written as part of the research project Nr WSC/2016/1 carried out as part of the statutory scientific.

Conflicts of Interest: The authors declare no conflict of interest.

\section{References}

1. Balat, M. Production of bioethanol from lignocellulosic materials via the biochemical pathway: A review. Energy Convers. Manag. 2011, 52, 858-875.

2. Jönsson, J.L.; Martín, C. Pretreatment of lignocellulose: Formation of inhibitory by-products and strategies for minimizing their effects. Bioresour. Technol. 2016, 199, 103-112.

3. Ahn, J.H.; Shinb, S.G.; Hwangb, S. Effect of microwave irradiation on the disintegration and acidogenesis of municipal secondary sludge. Chem. Eng. J. 2009, 153, 145-150.Klein, M.; Griess, O.; Pulidindi, I.N.; Perkas, N.; Gedanken, A. Bioethanol production from Ficus religiosa leaves using microwave irradiation. J. Environ. Manag. 2016, 177, 20-25.

4. Meinita, M.D.N.; Marhaeni, B.; Winanto, T.; Jeong, G.T.; Khan, M.N.A.; Hong, Y.K. Hong Comparison of agarophytes (Gelidium, Gracilaria, and Gracilariopsis) as potential resources for bioethanol production. J. Appil. Phycol. 2013, 25, 1957-1961.

5. Wu, F.C.; Wu, J.Y.; Liao Y.J.; Wang, M.Y.; Shih, I.L. Sequential acid and enzymatic hydrolysis in situ and bioethanol production from Gracilaria biomass. Bioresour. Technol. 2014, 156, 123-131.

(C) 2018 by the authors. Licensee MDPI, Basel, Switzerland. This article is an open access article distributed under the terms and conditions of the Creative Commons Attribution (CC BY) license (http://creativecommons.org/licenses/by/4.0/). 